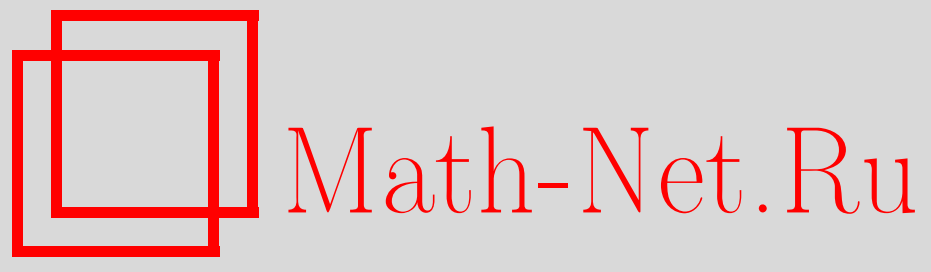

И. А. Михайлова, Об индексах избегаемости палиндромов, Матем. заметки, 2013, том 93, выпуск 4, 634-636 DOI: https://doi.org/10.4213/mzm9313

Использование Общероссийского математического портала Math-Net.Ru подразумевает, что вы прочитали и согласны с пользовательским соглашением http://www . mathnet.ru/rus/agreement

Параметры загрузки:

IP: 54.164 .48 .24

26 апреля 2023 г., 12:36:08

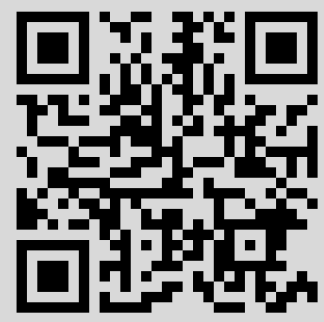




\section{Об индексах избегаемости палиндромов}

\section{И. А. Михайлова}

Пусть $\Sigma$ и $\Delta$ - два непустых конечных, но не обязательно различных, множества ( $а л ф а-~$ вита). Как обычно, через $\Sigma^{+}$(соответственно $\Delta^{+}$) обозначается множество всех непустых слов над алфавитом $\Sigma$ (соответственно $\Delta$ ). Отображение $h: \Delta^{+} \mapsto \Sigma^{+}$называется морфизмом, если для любых слов $p, q \in \Delta^{+}$выполняется равенство $h(p) h(q)=h(p q)$. Будем говорить, что слово $u \in \Sigma^{+}$избегает слово $p \in \Delta^{+}$, если для любого морфизма $h: \Delta^{+} \mapsto \Sigma^{+}$ слово $h(p)$ не является подсловом слова $u$. Слово $p$ называется $k$-избегаемым, если существует бесконечная последовательность слов $u_{i}$ над некоторым $k$-буквенным алфавитом такая, что каждое слово $u_{i}$ избегает $p$. Наконец, слово $p$ избегаемо, если существует число $k$ такое, что $p$ является $k$-избегаемым; в противном случае слово $p$ неизбежно. Наименьшее число $k$ такое, что слово $k$-избегаемо, называется индексом избегаемости этого слова. Для произвольного избегаемого слова задача нахождения его индекса избегаемости не решена, открытым остается вопрос и о сложности этой задачи (см. обзор [1]). Для слов некоторых типов удается получать те или иные верхние оценки на индекс избегаемости. Так, например, Петров [2] показал, что индекс избегаемости произвольного полного слова не превосходит 4 (полным называется слово, в котором каждая буква встречается дважды и для любых различных букв $x$ и $y$ в этом слове слова $x y$ и $y x$ являются его подсловами). В данной заметке указан еще один естественный класс слов, для которых имеется абсолютная (т.е. не зависящая от числа букв) оценка индекса избегаемости.

Напомним, что слово $p=a_{1} a_{2} \ldots a_{n}$ называется палиндромом, если оно совпадает со своим зеркальным образом $\overleftarrow{p}=a_{n} \ldots a_{2} a_{1}$. Мы докажем следующее утверждение.

Теорема 1. Индекс избегаемости произвольного избегаемого палиндрома не зависит от числа букв, входящих в запись этого слова, и не превосходит 16.

Избегаемый палиндром $a b a c b d b c a b a$ не принадлежит ни к одному из рассматривавшихся ранее классов избегаемых слов и о его индексе избегаемости ничего не было известно.

Для доказательства утверждения нам понадобятся дополнительные сведения об избегаемых словах.

1. Свободные стирания. Пусть $u$ - слово над алфавитом $\Sigma$. Пара подмножеств $B, C \subseteq \Sigma$ называется связкой в слове $u$, если для любого двухбуквенного подслова $x y$ слова $u$ выполняется $x \in B \Leftrightarrow y \in C$. Множество $A \subseteq B \backslash C$ называется свободным в слове $u$. Удаление из слова $u$ всех букв из свободного множества $A$ будем называть свободным стиранием и обозначать через $\delta_{A}$, а результат его применения к слову $u$ - через $u_{A}$. Последовательность $\delta_{A_{1}}, \delta_{A_{2}}, \ldots, \delta_{A_{k}}$ называется последовательностью свободных стираний, если $\delta_{A_{1}}-$ свободное стирание в слове $u, \delta_{A_{2}}-$ свободное стирание в слове $u_{A_{1}}$ и т.д.

Следующая теорема [3] поясняет связь между избегаемыми словами и свободными стираниями в словах.

Теорема 2. Слово и является неизбежным тогда и только тогда, когда существует последовательность свободных стираний такая, что результат ее применения к слову и - пустое слово.

Зафиксируем слова $u \in \Sigma^{+}, p \in \Delta^{+}$и морфизм $h: \Delta^{+} \mapsto \Sigma^{+}$такие, что $h(p)-$ подслово слова $u$. Рассмотрим множество $D \subseteq \Sigma$ и обозначим множество $\left\{x \in \Delta \mid h(x) \in D^{+}\right\}$ через $\bar{D}$. Построим новый морфизм $h_{D}:(\Delta \backslash \bar{D})^{+} \mapsto(\Sigma \backslash D)^{+}$по правилу $h_{D}(x)=(h(x))_{D}$. Тогда будет верна следующая лемма [4].

DOI: $10.4213 / \operatorname{mzm} 9313$ 
ЛЕмма 1. Пусть $u=u_{1} h(p) u_{2} \in \Sigma^{+}$для некоторого нестирающего морфизма $h$ и слова $p \in \Delta^{+}$, множество $D \subseteq \Sigma$ свободно в слове $u$. Тогда множество $\bar{D}$ свободно в слове $p$ и

$$
h_{D}\left(p_{\bar{D}}\right)=(h(p))_{D}
$$

2. Доказательство теоремы 1. Построим последовательность слов над алфавитом из 16 букв, которая является частным случаем конструкции, предложенной Сапиром в [4]. Рассмотрим матрицу $P$ размера $16 \times 4$ :

$$
P=\left(\begin{array}{cccc}
1 & 1 & 1 & 1 \\
2 & 1 & 2 & 1 \\
3 & 1 & 3 & 1 \\
4 & 1 & 4 & 1 \\
\vdots & \vdots & \vdots & \vdots \\
1 & 4 & 1 & 4 \\
2 & 4 & 2 & 4 \\
3 & 4 & 3 & 4 \\
4 & 4 & 4 & 4
\end{array}\right), \quad P_{\Sigma}=\left(\begin{array}{cccc}
a_{11} & a_{12} & a_{13} & a_{14} \\
a_{21} & a_{12} & a_{23} & a_{14} \\
a_{31} & a_{12} & a_{33} & a_{14} \\
a_{41} & a_{12} & a_{43} & a_{14} \\
\vdots & \vdots & \vdots & \vdots \\
a_{11} & a_{42} & a_{13} & a_{44} \\
a_{21} & a_{42} & a_{23} & a_{44} \\
a_{31} & a_{42} & a_{33} & a_{44} \\
a_{41} & a_{42} & a_{43} & a_{44}
\end{array}\right) .
$$

Обозначим через $\Sigma$ множество букв $\left\{a_{i j} \mid 1 \leqslant i, j \leqslant 4\right\}$. Матрица $P_{\Sigma}$ получена заменой элементов в матрице $P$ на буквы из $\Sigma$ по правилу: числу $i$, стоящему в $j$-м столбце, соответствует буква $a_{i j}$. Для каждого $1 \leqslant j \leqslant 4$ обозначим $\left\{a_{i j} \mid 1 \leqslant i \leqslant 4\right\}$ через $\Sigma_{j}$, тогда $\Sigma=\bigcup_{j=1}^{4} \Sigma_{j}$

Обозначим через $B_{i}$ слово, записанное в $i$-й строке матрицы $P_{\Sigma}$. Слова $B_{i}$, где $1 \leqslant i \leqslant 16$, называются блоками и обладают следующими очевидными свойствами [4].

Лемма 2. В каждом блоке все буквы различны, на $j$-й позииии стоит буква из $\Sigma_{j}$. Два различных блока не содержат одинаковых двухбуквенных подслов.

Рассмотрим морфизм $\gamma: \Sigma^{+} \mapsto \Sigma^{+}$такой, что $\gamma\left(a_{i j}\right)=B_{(i-1) 4+j}$. Покажем, что каждое из слов $\gamma^{m}\left(a_{11}\right)$, где $m \geqslant 0$, избегает все избегаемые палиндромы.

Предположим противное: существует (наименьшее) число $m$ такое, что для некоторого избегаемого палиндрома $p \in \Delta^{+}$и морфизма $h: \Delta^{+} \mapsto \Sigma^{+}$слово $h(p)$ является подсловом слова $\gamma^{m}\left(a_{11}\right)$. Без ограничения общности можно считать, что $h(p)=\gamma^{m}\left(a_{11}\right)$. Пусть $x y-$ некоторое двухбуквенное подслово слова $p$. Так как $p=\overleftarrow{p}$, то ух также будет его подсловом. В силу леммы 2 вторые индексы всех букв в каждом блоке возрастают от 1 до 4, поэтому $h(x)$ и $h(y)$ в слове $\gamma^{m}\left(a_{11}\right)$ могут располагаться только так, как показано на рис. 1.

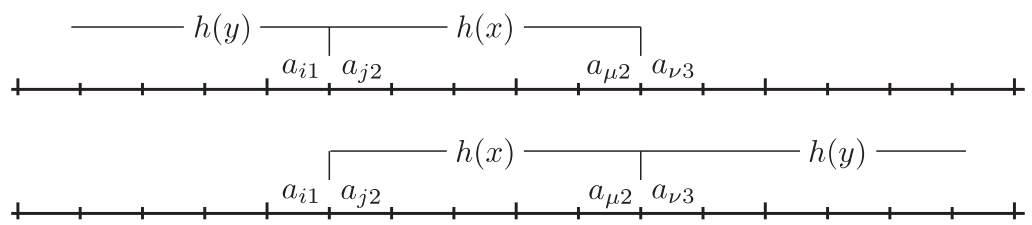

Рис. 1. Иллюстрация к доказательству теоремы 1.

Таким образом, число “границ" образов букв внутри блока не больше двух и, следовательно, в каждом блоке можно выбрать двухбуквенное подслово, которое для любой буквы либо лежит внутри образа этой буквы, либо не пересекается с ним. Зафиксируем некоторый набор таких двухбуквенных подслов и будем называть их маркерами. По лемме 2 каждое двухбуквенное подслово однозначно определяет блок. Каждому маркеру сопоставим новую букву $t_{B}$ и обозначим множество этих букв через $T$. 
Определим морфизм

$$
\bar{h}: \Delta^{+} \mapsto(\Sigma \cup T)^{+},
$$

где $\bar{h}(x)$ - это слово $h(x)$, в котором все маркеры заменены на соответствующие буквы из $T$. Рассмотрим слово

$$
\bar{h}(p)=P_{1} t_{1} Q_{1} P_{2} t_{2} Q_{2} \ldots P_{\ell} t_{\ell} Q_{\ell},
$$

где $P_{i}\left(Q_{i}\right)$ - префикс (суффикс) соответствующего блока. Следуя [4], будем называть это слово квази-целым (поскольку целыми в [4] назывались слова из $\Sigma^{+}$, которые можно разбить на блоки). В [4] было показано, что последовательность стираний $\delta_{\Sigma_{1}}, \delta_{\Sigma_{2}}, \delta_{\Sigma_{3}}, \delta_{\Sigma_{4}}$ является свободной в любом квази-целом слове. Результат применения этой последовательности к слову $\bar{h}(p)$ - это слово $\Lambda=t_{1} t_{2} \ldots t_{\ell} \in T^{+}$.

По лемме 1 последовательность $\delta_{\Sigma_{1}}, \delta_{\Sigma_{2}}, \delta_{\Sigma_{3}}, \delta_{\Sigma_{4}}$ порождает последовательность свободных стираний в слове $p$, результат применения которой обозначим через $p^{\prime}$. Заметим, что после стирания некоторого множества букв в палиндроме в результате снова получится палиндром. Определим морфизм $\varphi: T^{+} \mapsto \Sigma^{+}$по правилу

$$
\varphi\left(t_{B}\right)=a_{i j}, \quad \text { если } \quad \gamma\left(a_{i j}\right)=B .
$$

Тогда легко видеть, что

$$
\varphi\left(t_{1} t_{2} \ldots t_{\ell}\right)=\gamma^{m-1}\left(a_{11}\right) .
$$

По лемме 1 образ палиндрома $p^{\prime}$ при некотором морфизме является подсловом $\Lambda$ и, следовательно, слово $\varphi(\Lambda)=\gamma^{m-1}\left(a_{11}\right)$ не избегает $p^{\prime}$. Напомним, число $m$ было выбрано таковым, что $\gamma^{m-1}\left(a_{11}\right)$ избегает все избегаемые палиндромы. Откуда получаем, что слово $p^{\prime}$ неизбежно, и по теореме 2 слово $p$ также будет неизбежно, что приводит к противоречию и завершает доказательство теоремы.

\section{СПИСОК ЦИТИРОВАННОЙ ЛИТЕРАТУРЫ}

[1] J. D. Currie, Theoret. Comp. Sci., 339:1 (2005), 7-18. [2] А. Н. Петров, Матем. заметкu, 44:4 (1988), 517-522. [3] D. R. Bean, A. Ehrenfeucht, G. F. McNulty, Pacific J. Math, 85:2 (1979), 261-294. [4] M. V. Sapir, Combinatorics on Words with Applications, LITP Report 32, Université Paris 7, 1995.

\section{И. А. Михайлова}

Поступило

Уральский федеральный университет,

г. Екатеринбург

E-mail: inna.mikhaylova@gmail.com 